\title{
ISOMORFISMO INSTITUCIONAL EN EL AMBIENTE ACADÉMICO. CASO: ISO IWA-2
}

CARMEN CECILIA DAZA VILLADIEGO*

\section{RESUMEN}

En este trabajo se analizan aspectos de la teoría sobre el concepto de Isomorfismo Institucional, con miras a encontrar vías de reflexión frente a la alternativa de adopción y adaptación de la Norma ISO 9000, en su versión de IWA-2, en el ámbito académico. Se utiliza como marco teórico, por una parte, la teoría institucional y el neo-institucionalismo en los estudios organizacionales para proporcionar un marco conceptual consistente y apropiado para el estudio de la influencia de los factores del entorno en la función organizacional, que de alguna manera, impulsan el isomorfismo; sobre éste se hace un recorrido por la teoría y los tipos de isomorfismo en los que sucumben las organizaciones. A manera de conclusiones, se exponen algunas consideraciones que develan un marco normativo universitario institucionalizado que condiciona la búsqueda de referentes de calidad académica a través del isomorfismo mimético.

Palabras clave: Neo-institucionalismo, Isomorfismo Institucional, Mecanismos Isomórficos, ISO 9000 - IWA 2, Calidad académica

Clasificación JEL: B52, E11, L15, I21, I23

\begin{abstract}
This article analyzes several aspects about the concept of Institutional Isomorphism theory in order to find paths of reflection towards the alternatives for adopting and adapting the ISO 9000 normative on its IWA-2 version within the academic ambit. Institutional Isomorphism Theory and Neo-Institutionalism serve as theoretical frameworks in organizational studies in order to provide a consistent

* PhD (c) en Administración. Profesora Investigadora Pontificia Universidad Católica del Ecuador (Quito - Ecuador). Correo Electrónico: cdazav@puce.edu.ec
\end{abstract}


and appropriate base for studying the influence of environmental factors in the organizational function that somehow, motivates isomorphism. Based on this, we travel along the theory and types of isomorphism in which organizations succumb. Through the study of predominate isomorphic mechanisms, it is concluded that the contemporary university regulatory framework tends to pursue academic quality through the application of mimetic isomorphism.

Key words: Neo-institutionalism, Institutional Isomorphism, Isomorphic mechanisms, ISO 9000 - IWA 2, Academic quality

JEL Classification: B52, E11, L15, I21, I23

\section{INTRODUCCIÓN}

La Universidad es considerada por muchos, como un espacio privilegiado del ejercicio del análisis, la crítica de los fenómenos sociales y la búsqueda de propuestas de solución y cambio. Tiene una relativa autonomía institucional, y de acuerdo con ella, puede incrementar o disminuir su opción de ser permeable y sensible a las necesidades de la sociedad en su conjunto; puede modular su capacidad de respuesta a ella en la generación, desarrollo y aplicación de conocimientos dentro de los procesos de cooperación técnica, asesoramiento, elaboración de propuestas de intervención y de mejoramiento de servicios, a otras instituciones o grupos sociales.

Han sido fines de la Universidad a lo largo del siglo XII, el aumento del caudal del saber humano, y el desarrollo del espíritu de corporación, con el fin de defender los intereses comunes ${ }^{1}$. Frente a los desafíos marcados por la era de la calidad y del conocimiento, está llamada a satisfacer su vocación crítica en el aporte a un desarrollo evolutivo de la sociedad en el sentido ético-político de la formación humana.

Parece sin embargo, que la incorporación del criterio de calidad en la articulación de la educación superior como sistema, está ganando espacio frente a la generación de conocimiento propiamente, en virtud de las presiones de la competitividad, en el juego de la legitimación, y de la posesión de certificaciones internacionales y de acreditación institucional.

\footnotetext{
${ }^{1}$ En el siglo XII, el movimiento corporativo que dio lugar a la formación de los gremios, cofradías y hansas de artesanos y mercaderes, al reunir a los intelectuales, hizo surgir la corporación de profesores y alumnos: la universitas scholarum (21).
} 
En este sentido, en el ámbito de los procesos de certificación internacional de la calidad, se están generando condiciones propicias para establecer organizaciones educativas similares, lo que devela una marcada tendencia hacia el isomorfismo, en la manera y formas de normar actividades y en la búsqueda de legitimidad, esto es, la aceptación de la organización por parte de su entorno, lo cual resulta vital para la supervivencia y éxito organizativo.

Este trabajo propone analizar aspectos de la teoría sobre el concepto de isomorfismo institucional con miras a encontrar vías de reflexión frente a la alternativa de adopción y adaptación de la Norma ISO 9000, en su versión de IWA-2 (1), en el ámbito académico. Se utiliza como marco teórico, por una parte, la Teoría Institucional y el Neo-institucionalismo en los estudios organizacionales, por proporcionar un marco conceptual consistente y apropiado para el estudio de la influencia de los factores del entorno en la función organizacional, que de alguna manera, impulsan el isomorfismo; sobre éste se hace un recorrido por la teoría y los tipos de isomorfismo en los que sucumben las organizaciones. A manera de conclusiones, se cuestiona si la adopción de prácticas de normatividad en el ambiento universitario, para efectos de competitividad académica, develan un marco normativo institucionalizado ecléctico del sistema de educación superior, lo que condiciona la búsqueda de referentes a través del isomorfismo.

\section{INSTITUCIONALISMO Y NEO-INSTITUCIONALISMO}

Según una definición enciclopédica, el institucionalismo corresponde a una corriente del pensamiento económico y social, iniciada en Estados Unidos a finales del siglo XIX, cuyo objetivo era el análisis empírico de las instituciones propias del sistema capitalista. La corriente institucionalista, con Veblen ${ }^{2}$ (2) como su fundador, en el campo de la economía, concibe éste como una ciencia social al servicio de la humanidad; sitúa el cambio institucional en el centro de la investigación económica, enfocándose en el estudio de las necesidades de la gente real, no en las de los mercados abstractos, imaginados en los postulados de la teoría económica clásica y neoclásica.

El paradigma institucionalista desde un enfoque económico-social, concibe el mercado como un conjunto instituido de relaciones sociales

\footnotetext{
2 Thorstein Bunde Veblen (1857-1929) fue fundador de la corriente institucionalista en las ciencias sociales, junto con John R. Commons, Juhn Clark, Wesley Mitchell, en el pensamiento norteamericano, mientras que John Hobson representaba la escuela británica.
} 
y de reglas que determinan las condiciones de los intercambios (partes que pueden intervenir en el intercambio de productos, estructura de los derechos de propiedad, etc.), no son más que el resultado del ejercicio del poder por parte de instancias estatales o supraestatales.

La concepción neoclásica, en cambio, concibe el mercado como la institución dominante, como si fuera la causa suprema y todo lo demás el efecto. La posición neoliberal adopta el criterio de mercado como el único posible para guiar las políticas públicas y organizar la totalidad de la vida social. Para el enfoque institucionalista el mercado no es la causa, sino el efecto. La estructura de poder en la sociedad, esto es, las fuerzas reales operan detrás del mercado. Éste solamente refleja las reglas de juego establecidas previamente. Por ello, se aboga por la supeditación de la racionalidad del mercado a la racionalidad política democrática, la voluntad social.

La causa institucionalista, en definitiva, es modificar intencionalmente el sistema económico para introducir estructuras y prácticas que democraticen la participación ciudadana, tanto en el ámbito del trabajo, cuanto en el ámbito político. Ha habido un esfuerzo por elaborar una teoría del valor social distante del criterio de mercado; al menos, la primera generación de institucionalistas, desde Veblen (2) y Dewey ${ }^{3}$ (3), compartió el interés por el análisis de las instituciones, su evolución y el cambio institucional, junto con los problemas sociales y económicos. Para Veblen (2), la institución es una forma de 'hacer las cosas' (las instituciones tecnológicas) o de 'pensar cosas' (las instituciones ceremoniales). Esta primera generación, concibe las instituciones, desde una perspectiva crítica, como el conjunto de leyes, normas (instituciones formales), y el conjunto de valores, actitudes, hábitos mentales, tradiciones, convenciones sociales, etc. (instituciones informales) que regulan el comportamiento de las personas en la esfera social.

A partir de los años sesentas, un grupo de nuevos autores que configuran su pensamiento a partir de la obra de Veblen y del pensamiento de John Dewey, construyen su visión de la sociedad también inspirados en las experiencias libertadoras acaecidas en territorio norteamericano: los movimientos abolicionista y sufragista, de los derechos civiles, las protestas estudiantiles, el movimiento feminista, el movimiento

\footnotetext{
${ }^{3}$ John Dewey (1859-1952), El “instrumentalismo" de Dewey afirmaba que las cogniciones eran herramientas ideológicas o instrumentos con los que se confrontaban situaciones dadas. Mantenía que la mente era un instrumento para realizar los propósitos. Las ideas son, por tanto, armas tecnológicas de la mente, son elásticas y adaptables. (3).
} 
ecologista, las protestas por las políticas económicas neoliberales. Esta nueva corriente, los neo-institucionalistas ${ }^{4}$, aunque orientados hacia la política, consideraron además, que es el sistema de valores de la cultura que guía la economía y no los precios del mercado.

Desde un enfoque de la sociología de las organizaciones, que es de mayor interés en este trabajo, para esta nueva corriente los diferentes tipos de valores presentes en las instituciones son los que guían las conductas de los agentes sociales, por tanto, no hay forma de estudiar la realidad social si no es desde el punto de vista de los ideales humanos. Desde Veblen, el institucionalismo distingue entre valores instrumentales (ligados a la aplicación del conocimiento disponible a la resolución de los problemas en beneficio de la mayoría de la sociedad) y valores no instrumentales o ceremoniales (ligados a intereses cuya finalidad es la conservación del poder, estatus o privilegios). Se entiende, entonces, el cambio institucional como la sustitución de unos valores por otros, esto es, la modificación de las reglas de juego, la reconsideración de creencias y valoraciones anteriores, la sustitución de hábitos mentales y actitudes por otros, la revisión de rutinas establecidas, etc.

Una tercera vertiente de la economía institucional, cuya principal representación descansa en Willianson (4) y North (5) ${ }^{5}$, enmarcados dentro de la corriente neo-institucional, se distancia ampliamente de los planteamientos básicos de la teoría económica institucional de sus antecesores, de forma que se ha llegado a considerar de no institucional al nuevo institucionalismo (6).

Sin embargo, en el campo de la sociología y el análisis organizacional, tanto para la visión institucionalista como neo-institucionalista, las instituciones y las organizaciones componen el esquema básico del análisis institucional. En North (5), las instituciones son las reglas formales o informales que estructuran la interacción social, los incentivos y restricciones que dan

\footnotetext{
${ }^{4}$ Siguiendo a Gruchy (24) y Rutherford (26), forman parte de los llamados neo-institucionalistas: Clarence Ayres, Gunnar Myrdal, John Galbraith, Robert Hoxie, François Perroux, Adolph Lowe y Gerhard Colm.

${ }^{5}$ Esta nueva escuela de la economía institucional abarca gran variedad de corrientes, entre las que destacan la economía del derecho, que analiza los llamados costes de transacción y los derechos de propiedad, con Oliver Williamson (1985) (4), quien plantea que las transacciones pueden producirse a través del mercado o dentro de la propia empresa, implicando unos costes determinados, relativos tanto a la especificación del intercambio, como a los costes de hacer cumplir los acuerdos efectuados; y la nueva historia económica que contempla la historia como un proceso de evolución de las instituciones, con Douglas North $(5 ; 25)$, quien examina la naturaleza de las instituciones y las consecuencias del cambio institucional en el desempeño económico y social.
} 
forma a la interacción humana (el Sistema Judicial, el Catolicismo, los Derechos de Propiedad, el Código Familiar, el Mercado, El Sistema Educativo). Las organizaciones "son los jugadores" (Cortes, Juzgados, el Vaticano, la Familia, la Feria, la Bolsa de Valores, La Universidad) con distintas capacidades e intereses que operan con incentivos y restricciones definidos institucionalmente.

Por tanto, el Sistema Educativo, como institución, comprende el conjunto de normas jurídicas, los programas curriculares, la educación por niveles y grados, la educación no formal e informal, los establecimientos educativos, los organismos sociales (estatales o privados) con funciones educativas. Culturales y recreativas, los recursos humanos, tecnológicos, metodológicos, materiales, administrativos y financieros, articulados en procesos y estructuras para alcanzar los objetivos de la educación.

Ambos enfoques institucionalistas comparten, también, el escepticismo respecto de los modelos organizacionales basados en la idea de "actor racional". También, enfatizan las relaciones entre las organizaciones y sus ambientes, y el rol de la cultura como impulsora de la realidad organizacional. Siguiendo a North (5), es clave la distinción entre reglas y actores para entender los procesos de formación de preferencias y el cambio institucional, este último, fruto de la interacción entre instituciones y organizaciones en entornos de incertidumbre y competencia. Las instituciones reducen la incertidumbre y proporcionan una estructura a la vida diaria. North (5) distingue entre instituciones formales e informales. Las primeras, comprenden las leyes, los reglamentos y los procedimientos gubernamentales, mientras que las segundas, comprenden las ideas, las creencias, las actitudes y los valores de las personas, es decir, la cultura de una sociedad específica.

Dejando de lado un énfasis $\mathrm{u}$ otro, existe actualmente un intento de explicar la realidad social y la institución a través de tres aspectos esenciales en el proceso de institucionalización. Siguiendo a Scott (7), el proceso de institucionalización puede enmarcarse en tres mecanismos: regulativo, normativo y cognitivo.

El mecanismo regulativo, hace hincapié en que el conjunto de reglas expresas o implícitas de la organización son mecanismos clave para estandarizar e institucionalizar un esquema de comportamiento, pero los individuos actúan según un cálculo de costo-beneficio y siguen las reglas según sus propios intereses. En el mecanismo normativo, el conjunto de normas y valores (que pueden o no ser impuestos desdeafuera) condicionan 
el actuar de los individuos, pero a la vez les favorece al proporcionar un clima donde la acción social puede desarrollarse libremente, siempre en el marco normativo vigente. Respecto del mecanismo cognitivo, defendido por la escuela neo-institucionalista, se basa tanto en aspectos simbólicos como estructurales para la conformación y sostenimiento de las instituciones.

Según esta perspectiva, resultado de las presiones ejercidas por estos mecanismos, las organizaciones tienden a ser cada vez más homogéneas dentro de su campo y la práctica del isomorfismo surge como una importante consecuencia, tanto de los procesos competitivos como de institucionalización.

En este sentido, diferentes autores (Weber (8), Parsons (9), Durkheim (10), Berger y Luckmann (11)) han puesto énfasis en diferentes aspectos, estructurales o simbólicos, que consideran clave para el proceso de institucionalización. Por otra parte, Meyer y Rowan (12), DiMaggio y Powell (13) hacen hincapié en los sistemas culturales y normativos que les son impuestos o que son adoptados por el individuo o por las organizaciones, y consideran que el rasgo distintivo de cualquier institucionalización es el isomorfismo, esto es, como se anotó anteriormente, la aparición de fuerzas sociales poderosas como la competición en un sector, que impulsan a las organizaciones a adoptar características semejantes entre sí. Todo ello conllevaría a encontrar la legitimidad de la organización en su entorno, lo que les permite, por una parte, la expansión o el éxito y, además, disminuir la incertidumbre, $\mathrm{y}$, por otra, lograr un mecanismo por el cual, mediante reglas y normas compartidas, se estandaricen y se sancionen las desviaciones sobre lo instituido, manteniéndose las organizaciones isomórficas con los mitos sociales vigentes y evitando cualquier forma de ilegitimidad.

Por su parte, Selznick (14) anota la diferenciación entre una organización institucionalizada de otra que no lo es. Él afirma que cuando una organización está "institucionalizada" tiende a asumir un carácter especial y logra una habilidad distintiva; pero, ante todo, remarca el sentido de que la institución es una organización que está inmersa en el conjunto de valores del ambiente en que opera, más allá del cumplimiento de los requisitos técnicos propios de su función específica. Entonces, reconoce como verdaderas instituciones a las organizaciones como las iglesias, los hospitales y las escuelas y universidades; en ellas, la lógica de la eficiencia subyace o, por lo menos, se combina con la lógica del ambiente simbólico de sus estructuras valorativas (historia, cultura, hábitos, valores, y otros "mitos racionalizados"). 
Al respecto, Meyer y Rowan (12) aceptan como mitos racionales institucionalizados todas aquellas normas, sistemas legales, las profesiones, los programas, las tecnologías y una variedad de otras estructuras racionales necesarias para la organización, como sus sistemas de producción, de I+D, de mercadeo, etc., que a la postre se constituyen en sistemas de creencias compartidas por la comunidad organizativa y que conducen a la organización al logro de sus metas de supervivencia.

\section{ISOMORFISMO INSTITUCIONAL}

Respecto de las formulaciones de Weber (8) sobre legitimidad, desde entonces llama la atención el síndrome del isomorfismo, como el mecanismo de homogeneización de las organizaciones que compiten en un mismo campo organizacional. Se señala, además, la presión que se desarrolla al interior del campo para que las organizaciones busquen, por un lado, disminuir el grado de diversidad entre ellas y, por otro, legitimidad en la adopción de innovaciones, que luego se pretende que homogenicen las estructuras en dicho campo organizacional.

Powell y DiMaggio $(15 ; 13)$, procuran explicar la homogeneidad tan sorprendente de formas y prácticas organizacionales señalando que en las etapas iniciales del ciclo de vida de un campo organizacional este muestra una diversidad de estructuras y conductas entre sus organizaciones, pero una vez que se llega a una situación de relativa estabilidad, se presenta un severo impulso hacia la homogeneización. Los autores se refieren como campo organizacional al conjunto de organizaciones que constituyen un área o campo de la vida institucional y en la que confluyen la totalidad de los actores internos y externos, que complementan y regulan su actividad.

En cuanto a la definición institucional o estructuración del campo organizacional, que legitima su determinación, se precisa superar cuatro etapas establecidas, como sigue: i) aumentar el grado de interacción entre las organizaciones en el campo; ii) propiciar el surgimiento de estructuras interorganizacionales de dominio y de patrones de coalición claramente definidos; iii) incrementar la carga de información de la que deben ocuparse las organizaciones que participan en el campo; y iv) desarrollar la conciencia entre los participantes de un conjunto de organizaciones de que actúan en una empresa común (13). Como se afirmó anteriormente, una vez que las organizaciones logran identificarse en una misma línea de empresa, brotan fuerzas dominantes que las tornan más similares, bajo un proceso de homogeneización, que muchos coinciden en denominar isomorfismo. 
Siguiendo el trabajo de Powell y DiMaggio (13), se argumenta sobre dos tipos de isomorfismo (12; 16): Competitivo, que hace hincapié en la competencia del mercado, el cambio en los nichos y las medidas de ajuste. De acuerdo con el argumento de Aldrich (17), las organizaciones también persiguen en su accionar el poder político y la legitimidad institucional, por lo que atribuyen la clasificación de isomorfismo Institucional.

Buscando describir más claramente el proceso de isomorfismo institucional, Powell y DiMaggio (13) identifican tres mecanismos isomórficos predominantes, a través de los cuales se difunden los efectos institucionales de un campo organizativo:

Isomorfismo Coercitivo: es el resultado de la presión formal o informal de organizaciones que tienen prevalencia sobre otras. Así, el cambio o adaptación organizacional puede ocurrir como una respuesta directa a una nueva legislación o regulación gubernamental, o normas impuestas por empresas matrices a las filiales, o por presiones de la competencia cuando aparecen nuevas tecnologías o productos sustitutos (18).

Isomorfismo Mimético: caracterizado por la imitación o copia de políticas, estrategias, estructuras, tecnologías, sistemas productivos, bienes, servicios y prácticas administrativas, en general, suficientemente probadas y exitosas en organizaciones similares. Es visto como una respuesta a la incertidumbre y ambigüedad organizacional (15).

Isomorfismo Normativo: Es visto como resultado de la profesionalización que instituye un conjunto de normas y procedimientos tipificados por una ocupación o actividad específica. Pueden definirse, por ejemplo, las condiciones y los métodos de trabajo para controlar la producción, de modo de establecer una base cognitiva común que legitime la autonomía profesional. Puede lograrse por dos medios: a través de los cursos de profesionalización de la educación formal y por medio de las redes de relacionamiento dentro de la misma profesión, que pueden darse fuera de la organización en asociaciones profesionales, o dentro de la misma organización (15).

En ese orden de ideas, a partir de las teorías sobre isomorfismo presentadas, a continuación se hará alusión al conjunto de normas que conforman la Norma ISO 9001 IWA-2, como un proceso de institucionalización y legitimación de la práctica administrativa y académica en la universidad. 


\section{NORMATIVIDAD ISO 9001 IWA-2 ${ }^{6}$}

En el marco de la práctica del isomorfismo, se toma como referencia la Norma ISO IWA-2: International Workshop Agreement - Sistema de Gestión de la Calidad - que constituye una guía general para la aplicación de ISO 9001 y mejora de los procesos en las organizaciones que ofrecen servicio educativo ${ }^{7}$. La guía es proporcionada para auxiliar a las organizaciones educativas a relacionar los conceptos sobre sistemas de gestión de la calidad de las Normas ISO 9000 (1) con el quehacer educativo.

Las Normas ISO de la Serie 9000 (1) son un conjunto de normas y directrices internacionales para la gestión de la calidad. En sus primeras versiones, desde 1987, orientadas a entornos industriales, han logrado una importante difusión en todos los sectores empresariales como modelo para el desarrollo e implantación de Sistemas de Gestión de la Calidad SGC.

La familia de Normas ISO 9000 (1) vigentes, está constituida por tres normas básicas:

- ISO 9000 (1): Sistemas de Gestión de la Calidad SGC- Principios y Vocabulario;

- ISO 9001: Sistemas de Gestión de la Calidad SGC- Requisitos de Implementación;

- ISO 9004: Sistemas de Gestión de la Calidad SGC- Requerimientos para la Mejora Continua.

La norma ISO 9001 determina los requisitos del SGC de una organización para demostrar su capacidad de satisfacer las necesidades de los clientes y puede utilizarse para su aplicación interna por las organizaciones para certificación o con fines contractuales, mientras que la norma ISO 9004 proporciona recomendaciones sobre las mejoras del desempeño en las organizaciones y se sugiere como guía para perfeccionar el sistema, en su conjunto. La revisión de ambas normas se basa en los principios de gestión de la calidad que están recogidos en la norma ISO 9000 (1) y su

\footnotetext{
6 La responsabilidad de revisar el acuerdo del IWA cada tres años, es del organismo miembro designado por el Consejo de Gestión Técnica de la ISO y deben ser aprobadas por este, el cual decide si este acuerdo se confirma por otro período de tres años, transfiriéndolo a un organismo técnico de ISO para su revisión o su remoción. La primera versión de IWA-2 cubrió el período 2000-2003. Luego de posteriores revisiones en México, Malaysia, Panamá, Irlanda y Corea del Sur, en el presente se dispone de la Norma ISO 9001 IWA-2 en su versión 2007.

${ }^{7}$ La Norma ISO IWA-1 es la guía general para la aplicación de ISO 9001 y mejora de los procesos en las organizaciones que ofrecen servicios de salud.
} 
estructura responde a la de un sistema de gestión de la calidad basado en procesos que pueden gestionarse mediante el Ciclo de Control Deming ${ }^{8} \mathrm{O}$ Ciclo de Mejora Continua o PDCA

Las premisas que se han establecido en la Norma ISOIWA-2 son, comosigue?: i) Un plan de estudios puede especificar lo que se espera que sea aprendido por los educandos y cómo su aprendizaje será evaluado. Sin embargo, el plan de estudios por sí mismo no asegura que las necesidades y expectativas serán cubiertas si existen procesos deficientes en las organizaciones del campo educativo; ii) Todo sistema de gestión de la calidad es influenciado por los diferentes objetivos, por los diferentes métodos de enseñanza, y por prácticas administrativas diferentes, específicas de cada organización. Por consiguiente puede esperarse que los SGC varíen de una institución a otra; iii) El SGC que se diseñe al interior de cada organización educativa debe ser lo suficientemente sencillo y comprensible para alcanzar los objetivos de calidad esperados, los mecanismos esenciales de control que se formulen deben considerar la valoración del desempeño humano y la evaluación del proceso de enseñanza-aprendizaje, atendiendo la complejidad y dificultad de la exactitud en los procesos de medición, esenciales en todo sistema de gestión de la calidad.

\section{ISOMORFISMO Y NORMA ISO IWA-2}

Atendiendo los aspectos de legitimidad y de presiones competitivas que se generan al interior de los campos organizacionales, en el ámbito académico, y específicamente, para efectos del presente estudio, en el campo de la educación superior, los sistemas de gestión de la calidad de la serie ISO 9000 (1), originalmente diseñados para ambientes de tipo físico donde el desarrollo tecnológico ha permitido una fiabilidad y una disciplina en el control de los procesos de medición, presentan una tendencia muy marcada hacia la adopción de su estructura, con claras pretensiones de homogeneizar la actividad académica y administrativa en este campo organizacional.

El reconocimiento y el éxito probado en compañías de múltiples sectores a nivel nacional e internacional y los requerimientos del sistema educativo

${ }^{8}$ El Ciclo de Deming (22; 23), Ciclo de Shewhart o Ciclo PDCA (Plan, Do, Check, Act), fue introducido en Japón después de la II G. M., idealizado por Shewhart en la segunda década del siglo XX, y divulgado por Deming en 1950, quien efectivamente lo aplicó, logrando tornar más precisos y ágiles los procesos de negocio en el marco de la gestión de la calidad.

${ }^{9}$ Se han tomado, en base a la presentación de la Norma ISO IWA-2:2003 por parte del Foro Mundial de la Calidad INLAC 2005 
en cuanto a acreditación institucional con miras a promover el cambio hacia una mejora sustancial de la actividad académica de nivel superior, ha impulsado el isomorfismo mimético en las prácticas de gestión de la calidad, que implementadas acertadamente se constituyen en robustas plataformas sobre las cuales desarrollar procesos de autoevaluación y acreditación, resultado de las presiones directas o indirectas de los órganos gubernamentales pertinentes, por lo cual, la presencia del isomorfismo coercitivo se siente a nivel de todo el campo organizacional.

Respecto del isomorfismo normativo, la universidad latinoamericana se encuentra inmersa en un esfuerzo, conjuntamente europeo, por interpretar la Norma ISO 9001 a través del acuerdo IWA-2, con el propósito de homogeneizar los sistemas de gestión administrativa y académica, acatando la estructura general de la norma internacional, en medio de la complejidad de la organización universitaria y de su estructura de gobierno.

Complejidad que se evidencia en el caso del campo organizacional educativo como resultado de la profesionalización, y de la existencia de un conjunto de documentos legales o regulatorios que referencian a un SGC; terminología particular del sector, código de prácticas necesarias para la calidad en el sector, requisitos múltiples que los proveedores del SGC deben cumplir, necesidades de desarrollar esquemas de certificación y/o acreditación, etc. Condiciones que, a la postre, dificultan la realización de cambios radicales de origen endógeno, tal como afirman dentro de la teoría ecológica de las organizaciones Hannan y Freeman (19).

Por lo anterior, puede afirmarse que la gestión universitaria en los países latinoamericanos, aún delas diferencias reales de sus sistemas de educación superior, se encuentra embarcada en los mismos procesos de cambio, lo que puede interpretarse como producto de un proceso isomórfico de cambio social (20). Ante tales circunstancias, cabe preguntarse ¿en qué medida estos modelos de inducción del cambio pueden generar resultados similares y estabilizar el campo organizacional?

\section{CONSIDERACIONES FINALES}

Este artículo, tuvo como objetivo revisar la teoría institucionalista y neoinstitucionalista desde el enfoque organizacional, para lo cual se enfatizó sobre las características de los mecanismos isomórficos para promover el cambio organizacional. Siguiendo a Powell y DiMaggio (13), se revisó muy someramente el contenido de la práctica del isomorfismo normativo 
a través de la adopción de las estructuras de las Normas ISO 9001 e ISO IWA-2, en la organización universitaria que, como Selznick (14) señala, es considerada una organización institucionalizada.

Respecto del enfoque, de participación y formalización que caracterizan a las Normas ISO, el componente de control a través del sistema de medición, no sólo considera aspectos financieros, sino que también, y especialmente, considera el contexto en el que se realizan las actividades, tratando de orientar e influir en que el comportamiento individual y organizativo sea el más conveniente para alcanzar los objetivos de la organización. En este sentido, la extensión de los sistemas de calidad al mundo de los servicios suscita nuevos problemas aún no resueltos, en el establecimiento y la fiabilidad de las medidas para el control de la gestión en sí misma.

La manera en que los sistemas de control y de gestión de la calidad, pueden ser aplicados de manera eficaz y eficiente en una organización de servicios, está limitada fuertemente por la imaginación e ingenio de los individuos en la organización que tengan interés en lograr la excelencia. Las personas en la organización, responsables de prestar el servicio pudieran enfocarse en los procesos de tipo administrativo antes que en aquellos que determinan la calidad de la actividad principal del núcleo del servicio, que para el caso de la universidad, es lo académico.

La fina tarea de la docencia goza de un grado de libertad académica, difícil de medir y de juzgar. El carácter mismo de la institución: la autonomía en su gestión administrativa y especialmente académica, la hacen una organización muy peculiar, que exige mecanismos de control muy sofisticados, que valoricen la producción intelectual e investigativa en grado mayor a los aspectos de eficiencia, propiamente.

Como lo expresa Borrero (21):

"Las universidades son instituciones científicas, reacias, en muchos aspectos, a todo dictamen burocrático. Sus funcionarios y profesores son científicos y profesionales. Nada autoriza a sus directivos para regir en detalle la conducta investigativa y docente del profesorado, a la manera como un gerente o el jefe de un taller determina la acción cotidiana de empleados y trabajadores"

Sin embargo, cada vez más las instituciones universitarias están considerando que la Norma ISO IWA-2 les permitirá enfocarse más en los procesos sustantivos del quehacer educativo, es decir, aquellos directamente relacionados con la enseñanza y el aprendizaje. 
La cuestión que se pretende transferir al lector es: el marco normativo institucionalizado en el sistema de educación superior condiciona la búsqueda de referentes de calidad a través del isomorfismo mimético; en este sentido, ¿cuáles son las reales implicaciones de la estandarización del sistema educativo a nivel superior, a través de la tipificación de procedimientos establecidos bajo la normatividad internacional del sistema ISO 9000-IWA 2 (1), como la vía para alcanzar el nivel de calidad deseado que contribuya al fortalecimiento del sistema educativo universitario?

\section{REFERENCIAS BIBLIOGRÁFICAS}

1. International Organization for Standardization. International Organization for Standardization. http://www.iso.org. [En línea] 2003. [Citado el: 11 de Enero de 2011.] http://www.iso.org/iso/ home/standards/management-standards/iso_9000.htm.

2. Veblen, Thorstein. The Theory of the Leisure Class: an economic study of institutions. Dheli: Aakar Books, 1899.

3. Dewey, John. Logic: The Theory of Inquiry. New York : Holt, Rinehart and Winston, 1938.

4. Willianson, Oliver. The Economic Institution of Capitalism: Firms, Markets, Relational Contracting. New York: Free Press, 1985.

5. North, Douglass C. Institutions, Institutional Change and Economic Performance. Cambridge: Cambridge University Press, 1990.

6. Institutionalism as a school - A reconsideration. Klein, Philip A. 2, s.l.: Association for Evolutionary Economics, Junio de 1990, JEI - Journal of economic issues, Vol. 24, págs. 381-388.

7. Scott, Richard. Institutional Theory and Organizations. Londres: Sage Publications, 1995.

8. Weber, Max. Economía y Sociedad. México D.F.: Fondo de Cultura Económica, 1997.

9. Parsons, Talcott. Estructura y Proceso en la Sociedad Moderna. Madrid: Instituto de Estudios Políticos, 1968.

10. Durkheim, Emile. Educción y sociología. Bogotá: Linotipo, 1979.

11. Berger, Peter y luckmann, Thomas. La construcción social de la realidad. Buenos Aires: Amorrortu, 1997.

12. Institutionalized Organizations: Formal Structure as Myth and Ceremony. Meyer, John W. y Rowan, Brian. 2, s.l.: The University of Chicago Press, Setiembre de 1977, American Journal of Sociology, Vol. 83, págs. 340-363. 
13. Powell, Walter W. y Dimaggio, Paul J. El nuevo Institucionalismo en el análisis organizacional. México D. C. : Fondo de Cultuta Económica, 1999.

14. Institutionalism "Old" and "New". Selznick, Philip. 2, s.l.: Sage Publications, Inc. on behalf of the Johnson Graduate School of Management, Cornell University, Junio de 1996, Administrative Science Quarterly, Vol. 41, págs. 270-277.

15. The Iron Cage Revisited: Institutional Isomorphism and Collective Rationality in Organizational Fields. DiMaggio, Paul J. y Powell, Walter W. 2, s.l.: American Sociological Association, Abril de 1983, American Sociological Review, Vol. 48.

16. The Effects of Environmental Characteristics on the Structure of Hospital Clusters. Fernnell, Mary. 3, s.l.: Sage Publications, Inc. on behalf of the Johnson Graduate School of Management, Cornell University, Septiembre de 1980, Administrative Science Quarterly, Vol. 25, págs. 485-510.

17. Environments of Organizations. Aldrich, Howard y Pfeffer, Jeffrey. s.l.: Annual Reviews, 1976, Annual Review of Sociology, Vol. 2, págs. 79-105.

18. Pfeffer, Jeffrey. Organizations and organization theory. Boston: Pitman, 1982.

19. Hannan, Michael y Freeman, John. Organizational Ecology. Cambridge: Harvard University Press, 1993.

20. Meyer, John. The Impact of the Centralization of Educational Funding and Control on State and Local Organizational Governance. ublications, Institute for Research on Educational Finance and Governance, School of Education/CERAS Bldg., Stanford University. Stanford: Stanford University, 1979.

21. Borrero, A. Simposio permanente sobre la Universidad. Santa Fé de Bogotá: Universidad Javeriana, 2004.

22. Deming, William. Calidad, Productividad y Competitividad. Madrid: Díaz de Santos S.A., 1989.

23. - . La nueva economía. Madrid: Díaz de Santos S.A., 1997.

24. Gruchy, Allan. Contemporary Economic Thought: The contribution of neo-institutional economics. New York: The MacMillan Press Ltda., 1972.

25. North, Douglass. Understanding the Process of Economic Change. Princeton: Princeton University Press, 2005.

26. Rutherford, Malcolm. Understanding Institutional Economics: 19181929. 3, Cambridge: Cambridge University, 2000, Journal of the History of Economic Thought, Vol. 22, págs. 147-160. 


\section{BIBLIOGRAFÍA RECOMENDADA}

Chandler, A. (1962). Strategy and Structure: Chapters in the History of the American Industrial Enterprise, Cambridge, MA, MIT Press.

Cooper, R. (1995). When Lean Enterprises Collide: Competing through confrontation, Boston, Harvard Business Scholl Press.

Furubotn E. y Richter R. (1991). The New Institutional Economics: An Assesment. Texas, Texas A\&M University Press.

J. Drobak y J. Nye. (1997). The Frontiers of the New Institutional Economics, London, Academic Press

Perrow, Ch. (1991). Sociología de las Organizaciones, Madrid, Mc Graw Hill.

Prado, I. C. (2000). El Proceso de Mejora Continua en la Empresa, Madrid, Pirámide.

Scott, W. R. (1995). Institutions and Organizations. Sage Publications. Londres.

(1998). Multidimensional Model of Organizational Legitimacy: Hospital Surviving en Changing Institutional Environments. Cornell University, Administrative Science Quarterly, 43, 877-904 .

UNESCO. (1998). Declaración Mundial sobre Educación Superior en el siglo XXI: Visión y Acción. Conferencia Mundial sobre Educación Superior. Fecha de consulta: enero 10 de 2011. Disponible en: http:// www.unesco.org/education/educprog/wche/declaration_spa.htm. 